\title{
Laparoscopic Treatment of Adult Sigmoidorectal Intussusception Caused by a Mucinous Adenocarcinoma of the Sigmoid Colon: A Case Report
}

\author{
Seok Youn Lee, Won Cheol Park, Jeong Kyun Lee, Dong Baek Kang, Young Kim, Ki Jung Yun ${ }^{1}$ \\ Departments of Surgery and ${ }^{1}$ Pathology, Wonkwang Medical Center, Wonkwang University College of Medicine, Iksan, Korea
}

\begin{abstract}
Intussusception is a rare cause of intestinal obstruction in adult patients, but is common in children. In fact, it accounts for an estimated $1 \%$ of all cases of bowel obstruction in adults, although adult intussusception of the large intestine is rare. Sigmoidorectal intussusception, however, is a rare variety with few cases reported in the literature. A mucinous adenocarcinoma, a subtype of adenocarcinoma, is characterized by extracellular mucin production and accounts for between $5 \%$ and $15 \%$ of the neoplasms of the colon and rectum. Despite the general consensus supporting surgical resections for adult intussuceptions, controversy remains over whether intussuceptions should be reduced before resection. Most cases of colon intussusception should not be reduced before resection because they most likely represent a primary adenocarcinoma. However, prior reduction followed by a resection can be considered for the sigmoidorectal intussusception to avoid inadvertent low rectal cancer sugery. We experienced one case of sigmoidorectal intussusception caused by a mucinous adenocarcinoma of the sigmoid colon in a 79-year-old woman. Abdominal computed tomography demonstrated a sigmoidorectal intussusception. After the end-to-end anastomosis-dilator-assisted reduction, the patient underwent a laparoscopic oncological anterior resection under the impression that a sigmoidorectal intussusception existed. We report a successful laparoscopic anterior resection in a patient with an intussusception caused by a sigmoid malignant tumor.
\end{abstract}

\section{Keywords: Sigmoidorectal intussusception; Mucinousa adenocarcinoma}

\section{INTRODUCTION}

Intussusception is a relatively common disease in children, but it is a relatively rare disease in adults, and leading points in adults are different from those in children. In patients showing bowel obstruction, the cause has been reported to be intussusception in $1 \%$ of the patients, and cases with sigmoidorectal intussusception are not common [1]. Lipoma, adenoma, polyps, Meckel's diverticulum have been reported to be causes. Among malignant tumors,

Received: August 2, 2010 Accepted: October 7, 2010

Correspondence to: Won Chul Park, M.D.

Department of Surgery, Wonkwang Medical Center, Wonkwang University

College of Medicine, 344-2 Sinnyong-dong, Iksan 570-711, Korea

Tel: +82-63-859-1496, Fax: +82-63-855-2386

E-mail: parkwc@wonkwang.ac.kr

(C) 2011 The Korean Society of Coloproctology

This is an open-access article distributed under the terms of the Creative Commons Attribution NonCommercial License (http://creativecommons.org/licenses/by-nc/3.0) which permits unrestricted non-

commercial use, distribution, and reproduction in any medium, provided the original work is properly cited. adenocarcinomas have been reported to be a cause. Nonetheless, cases caused by the subtype of adenocarcinoma, a mucinous adenocarcinoma, showing relatively poor prognosis are very rare [2-4]. In adult intussusception, the principle is surgical resection of the invaginated intestine and the causative lesion. Nevertheless, surgery may be modified depending on the disease history of the patient and surgical findings $[1,2,5,6]$. In most reports, treatment of sigmoidorectal intussusception after the reduction of intussusception by a laparotomy has been reported. However, it is true that cases of successful laparoscopic treatments are rare [7].

The clinical symptoms and physical examination findings of patients are diverse. However, due to masses invaginated to the lower rectum, sigmoidorectal intussusception may be misdiagnosed as rectal cancer in the lower part based on a digital rectum examination; thus, for effective treatment, intussusceptions must be diagnoses accurately prior to surgery.

In addition, in the treatment of lower rectal cancer, despite remarkable improvements of laparoscopic techniques, complications in the urogenital system caused by injury of the autonomic system 
have been reported. In the past, it was difficult to diagnose the disease because of its low incidence. Recently, it could be accurately diagnosed through the use of ultrasonography and abdominal computed tomography.

We experienced a case in which the sigmoidorectal intussusception could be reduced to the proximal sigmoid colon through the anus in a patient admitted for the chief complaints of recurring pain in the abdomen from several months ago, hematochezia, and constipation. Lower rectal cancer was suspected at an outpatient clinic, but a mucinous adenocarcinoma was diagnosed by using pathologic examination. This case is reported, together with a review of the literature.

\section{CASE REPORT}

A 79-year-old female patient visited the outpatient clinic with the chief complaints of hematochezia associated with diarrhea from several month ago, tenesmus, and pain in the lower abdomen for colonoscopic examination. Because of insufficient bowel preparation and the abdominal pain of the patient becoming severe, abdominal computed tomography was performed, malignant tumors were suspected, and the patient was admitted to our hospital. In the past history and family history, no special findings were detected. At the time of admission, the blood pressure was 100/80 $\mathrm{mmHg}$, the temperature was $37.6^{\circ} \mathrm{C}$, and the pulse was 74 per minute; the patient was conscious. In cardiopulmonary auscultation, no special findings were detected, and in the abdominal area, mild tenderness was detected. A movable mass associated with hemorrhage was palpated in an area approximately $5 \mathrm{~cm}$ above the anal verge. Other than the above, no special findings were detected.

In the general peripheral blood test, leucocytes were $5,000 / \mu \mathrm{L}$, hemoglobin was $7.5 \mathrm{~g} / \mathrm{dL}$, and platelets were $248,000 / \mu \mathrm{L}$. In serum biochemistry tests, AST was $24 \mathrm{IU} / \mathrm{L}$, ALT was $23 \mathrm{IU} / \mathrm{L}$, ALP was $215 \mathrm{IU} / \mathrm{L}$, total bilirubin was $0.38 \mathrm{mg} / \mathrm{dL}, \gamma$-GTP was $6 \mathrm{IU} / \mathrm{L}$, total protein was $6.6 \mathrm{~g} / \mathrm{dL}$, albumin was $3.6 \mathrm{~g} / \mathrm{dL}$, BUN was $15.2 \mathrm{mg} / \mathrm{dL}$, creatinine was $0.52 \mathrm{mg} / \mathrm{dL}$, prothrombin time was 12.1 seconds (INR, 1.16), HBs Ag was negative, anti-HBs Ab was positive, anti$\mathrm{HCV}$ Ab was positive, and CEA (RIA) was elevated to $82.1 \mathrm{ng} / \mathrm{mL}$. In plain abdominal radiography, the retention of a large amount of feces could be detected. In anoscopic examination, the rectal inner wall was observed to be occluded by a mass in an area approximately $3 \mathrm{~cm}$ above the anal verge and to be covered with dark blood. Continuous hemorrhage from the surface was detected, and the mass was palpated to be relatively movable (Fig. 1). In abdominal computed tomography, an irregularly shaped mass in the sigmoid colon approximately $5 \times 3.8 \times 3.9 \mathrm{~cm}^{3}$ in size and associated with the intussusception from the mass to the rectum, was detected. In addition, along the invaginated mesentery, about 2 lymph nodes showing contrast enhancement were observed (Fig. 2).

Under general anesthesia, the patient was placed in the modified lithotomy position, the trendelenberg position, and bent with the right side downward. A 12-mm trocar was inserted into the vicinity of the umbilicus, a $12-\mathrm{mm}$ trocar was inserted into the right lower abdomen, a 5-mm trocar was inserted into the right upper abdomen, and an 11-mm trocar was inserted into the left lower abdomen. The sigmoid colon and the rectum were examined, and an intussusception of the sigmoid colon to the inside of the lower rectum was detected. Laparoscopic reduction of the invaginated colon was attempted using a laparoscopic grasper, but failed. Hence, a dilator for end-to-end anastomosis (EEA; $28 \mathrm{~mm}$ ) was inserted through the anus of patient, the mass was pushed up carefully, simultaneously reduction using a laparoscopic grasper was attempted, and the invaginated area could be readily reduced to the abdominal cavity. The reduced area of mass did not show necrotic findings, and except for mild hemorrhage in the vicinity of the appendiceal epiploica of the sigmoid colon, normal findings were shown (Fig. 3). The reduced mass was restored to the proximal sigmoid colon, and the infer mesenteric vessels were ligated. Subsequently, a laparoscopic anterior resection and primary anastomosis were performed, and surgery was completed. Histological examination was diagnosed a mucinous adenocarcimoma, and metastasis was detected in 10 lymph nodes (Fig. 4). In microscopic examination, infiltration by the adenocarcinoma and secretion of mucus could be observed; thus, it was diagnosed as a mucinous adenocarcinoma (Fig. 5). Three days after surgery, movement of the intestine of the patient was recovered, and diet was initiated. The patient had slight diarrhea symptoms, but recovered without other complications, and is presently under follow-up observation at our outpatient clinic.

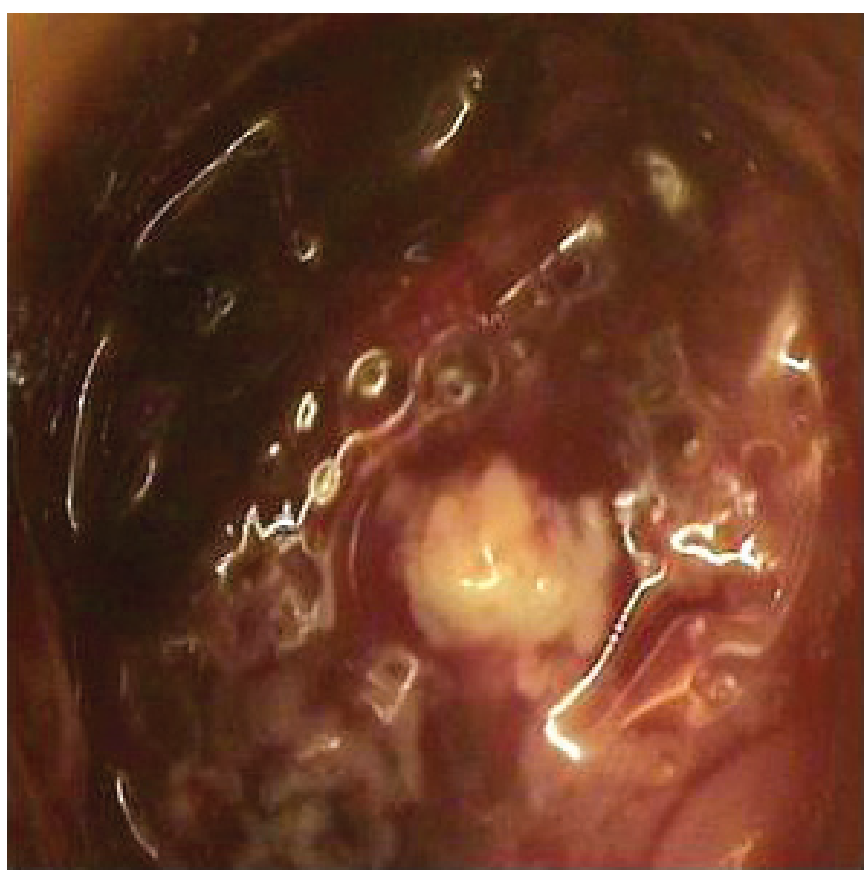

Fig. 1. The anoscopic finding shows a large, friable, and hemorrhagic mass lesion $3 \mathrm{~cm}$ from the anal verge and occupying the entire rectum. 


\section{Coloproctology Seok Youn Lee, et al.}
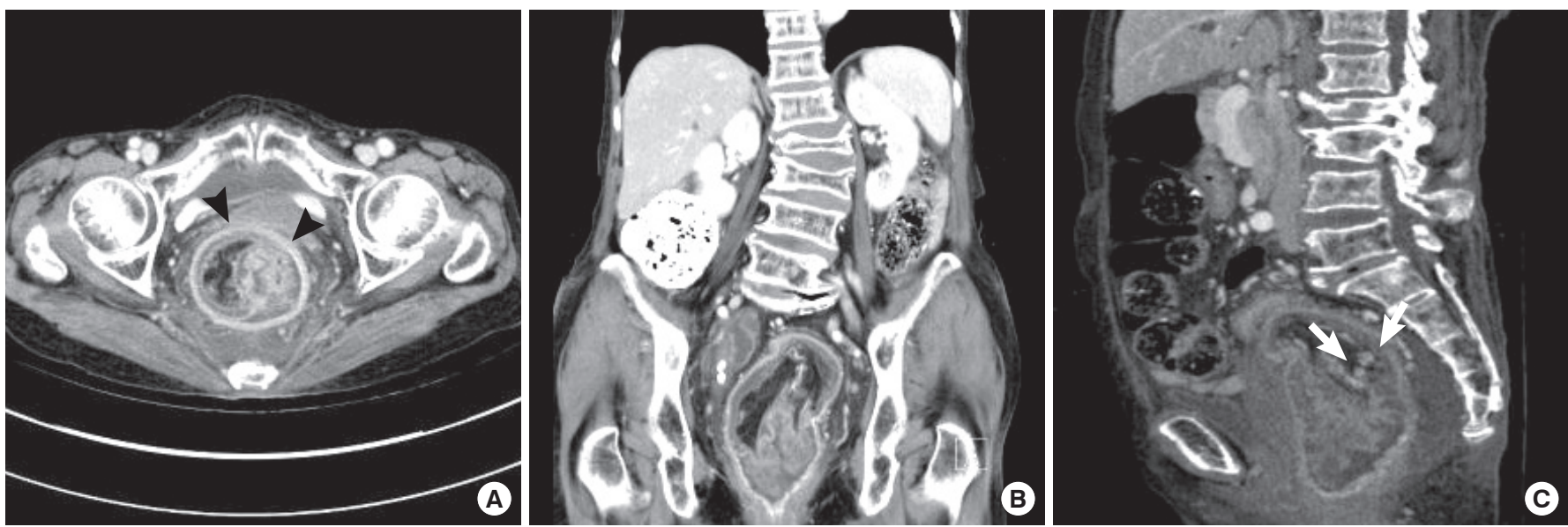

Fig. 2. CT shows an intussusception mainly from the proximal sigmoid colon invaginated into a rectum: (A) axial view, (B) coronal view and (C) sagittal view. Fat was seen within the intussusceptions (target sign, arrowheads), and about a $5 \times 4 \mathrm{~cm}$ sized irregularly shaped mass was seen in the rectum. Normal rectal wall was seen surrounding normal sigmoid lesion and small lymph nodes were seen within the invaginated mesentery (arrows).
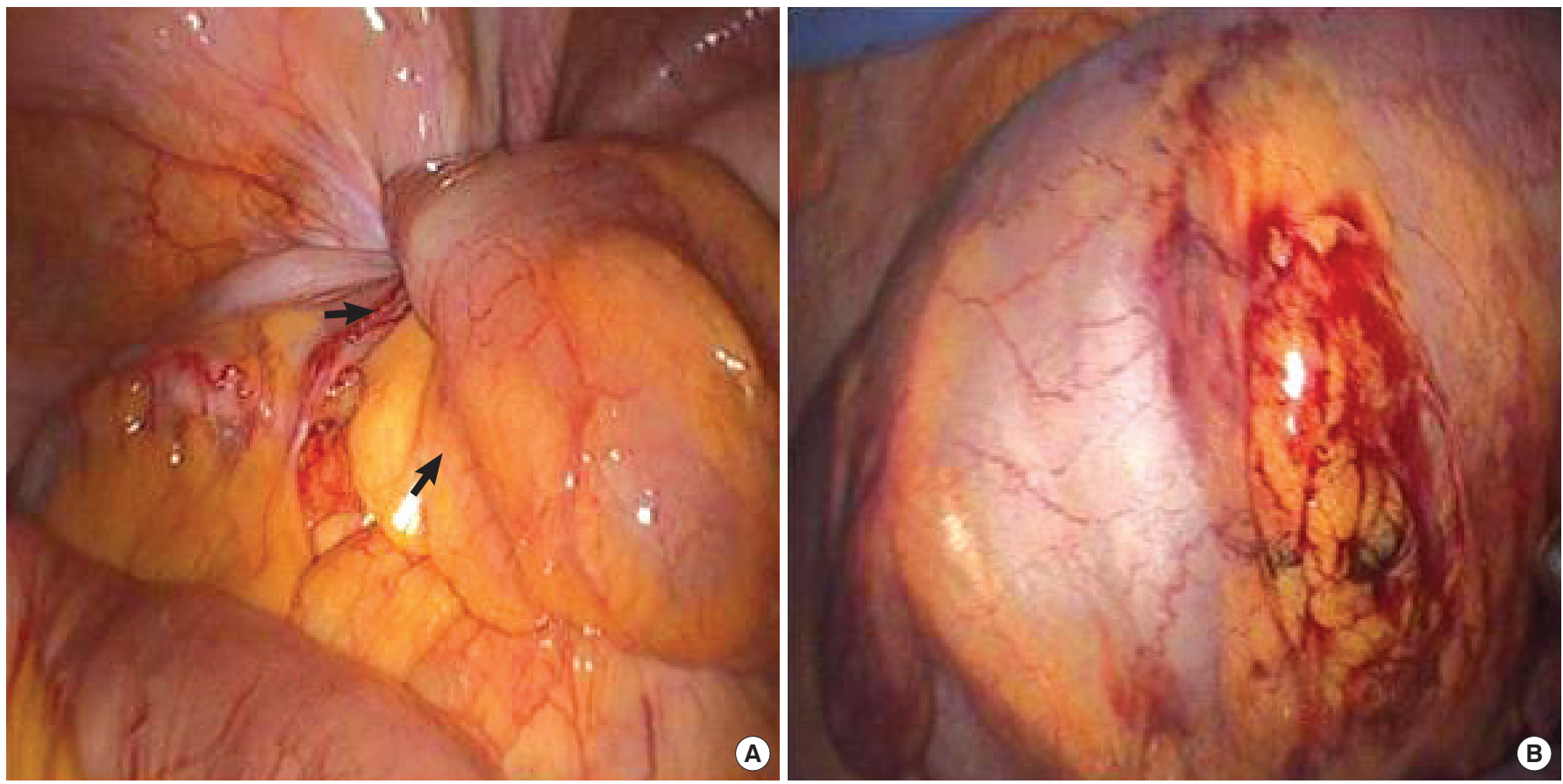

Fig. 3. (A) Laparoscopic view of the large sigmoidal mass intussuscepting into the rectum (arrows). (B) After sigmoid colon reduction, a mild hemorrhage was seen at the appendices epiploica of the sigmoid colon. There was no evidence of bowel necrosis or demarcation.

\section{DISCUSSION}

Intussusception was described by Barbette of Amsterdam in 1674 for the first time, and in the early 1700s, Velse performed surgery for adult intussusception successfully [6]. Afterward, in 1871, Sir Jonathan Hutchinson performed surgery on infants for the first time [8]. Intussusception is caused by the invagination of lesions, as well as the adjacent intestinal wall, to the inside of the distal bowel due to the peristalic movement of the intestine. The mesocolon and blood vessels are invaginated together and block the supply of blood flow, resulting in ischemic necrosis of the intestinal wall. Thus, it is a disease requiring early diagnosis and treatment.

Adult intussusception is generally caused by underlying diseases; on the other hand, in children, it develops without cause in most cases [5]. According to several reports, adult colonic intussuscep- 


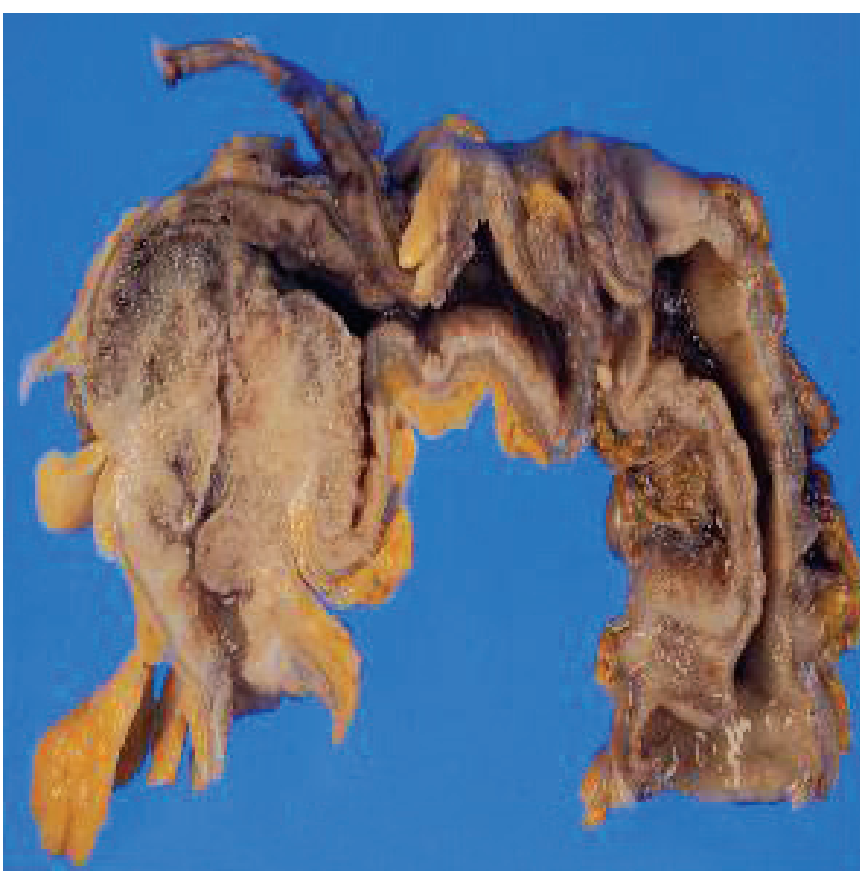

Fig. 4. The gross specimen included a $7 \times 8 \mathrm{~cm}^{2}$ mass, thought to be the lead point of the intussusception, in the sigmoid colon.

tion is caused by malignant tumors in $64 \%$ to $87 \%$ of patients and by benign tumors in $29 \%$ to $33 \%$ of patients $[5,6,9,10]$. Nagorney et al. [5] reported adult intussusception according to its location. Approximately $38 \%$ of intussusceptions have been reported to develope in the small intestine, $6 \%$ of intussusceptions are ileo-cecal types and ileo-colic types, and approximately $50 \%$ of intussusceptions develop in the colon. Among colonic intussusceptions, approximately $70 \%$ develop in the right colon, and intussusceptions in the left colon and the sigmoid colon are rare. Approximately $65 \%$ of colonic intussusceptions are reported to be associated with malignant tumors; hence, attention should always be paid to that possibility during diagnosis and surgery.

It has been reported that in regard to the most common causes of colonic intussusception, benign tumors are lipomas, leiomyomas, adenomatous polyps, endometriosis that developed in the appendix, and the anastomosis area of previous surgery, and malignant tumors are adenocarcinomas, lymphomas, lymphosarcomas, and leiomyosarcomas [6]. In addition, although rare, intussusception caused by a lymphangioma in the abdominal cavity has been reported [4]. In Korea, similarly, several studies on intussusception have been reported; nonetheless, it is true that reports on sigmoidorectal intussusception are rare, and most are reports on benign adenomatous polyps $[11,12]$. In Korea, intussusception cases caused by mucinous adenocarcinomas and treated by using a laparoscopic procedure have not been reported yet. In addition, the incidence of the subtype of adenocarcinoma, the mucinous adenocarcinoma, has been reported to be relatively rare, to occur preferentially in young age groups, and at the time of diag-

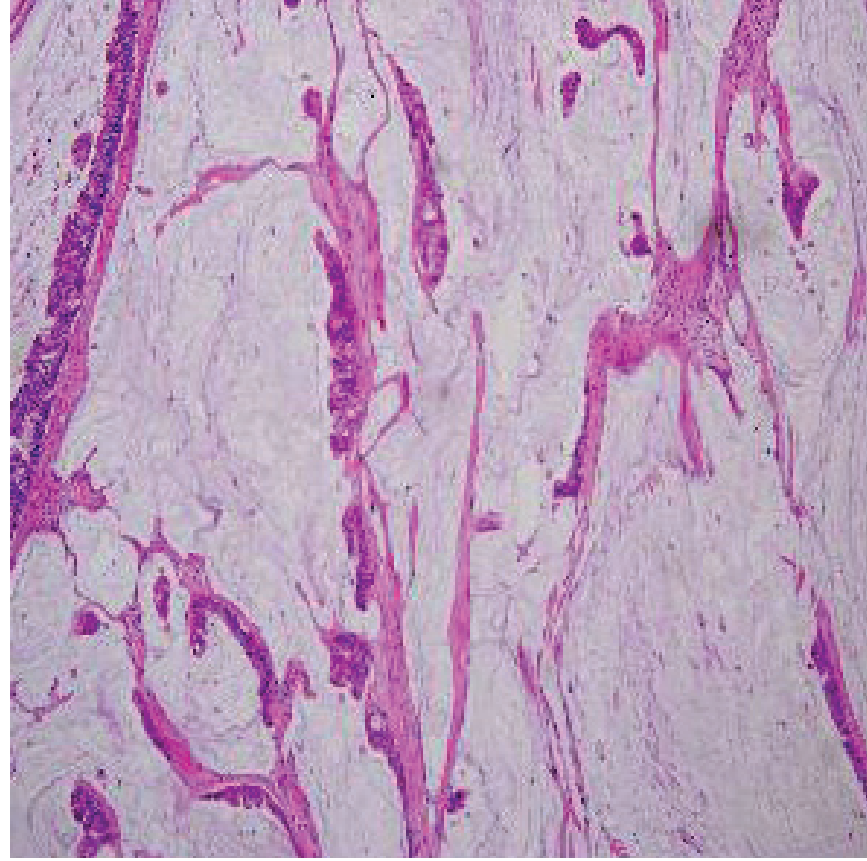

Fig. 5. Histopathological features were consistent with a mucinous carcinoma. Tumor cells scattered in pools of mucus are demonstrated $(\mathrm{H}$ $\& \mathrm{E}, \times 100)$.

nosis, to have progressed locally in many cases, and different from an adenocarcinoma, it is developed in the proximal colon, and its prognosis is poor $[13,14]$.

The subjective symptoms of patient are generally very diverse and are, thus, vague in many cases; hence, the preoperative diagnostic rate is $20-25 \%$, and prior to surgery, it may be diagnosed as ileus, abdominal masses, gastrointestinal hemorrhage, etc. The most common symptom is abdominal pain, and rather than acute pain, it is regular and intermittent abdominal pain for a long time. Vomiting and hematochezia are the second common symptoms. Azar and Berger [1] reported that nausea, vomiting, abdominal pain and hematochezia manifested during bowel obstruction are common. In addition, it may be manifested as weight loss, fever, constipation, diarrhea, abdominal masses, etc., and only repeated intermittent abdominal pain may be presented for several years in some cases.

On physical examination, abdominal masses are palpated in 24$42 \%$ of the cases, and in most cases, they are mobile and can be palpated when it is painful $[5,15]$. Laboratory findings are normal or nonspecific in most cases, and anemia and a slightly increased number of leucocytes may be detected [16]. Since sigmoidorectal intussusception is very rare, it is difficult to diagnosis only by physical examination, and if it is invaginated to the lower rectum and associated with continuous hemorrhage as was our case, it may be readily misdiagnosed as lower rectal cancer. It is difficult to diagnose intussusception clinically in many cases, and it is diagnosed primarily by using radiological tests. In plain abdominal radiogra- 
phy, findings of ileus and soft tissue density may be suspected to be an intussusception; nonetheless, such findings are not specific, and colonic intussusception can be diagnosed by specific findings, such as the filling defect within a barium study, an occlusion, a coil-spring shape, etc., but the entero-enteric type cannot be diagnosed. As the applications of abdominal ultrasonography and abdominal computed tomography become generalized, intussusception can be diagnosed more readily. In abdominal computed tomography, if target signs or doughnut signs are shown on a cross section of the intussusception, and pseudo-kidney signs are shown on transverse sections, it can be diagnosed. In abdominal computed tomography, it can be diagnosed by concentric rings associated with low contrast lesions or by the enlargement of small intestine segments $[15,17]$. Recently, if the cause is a tumor as in our case, whether malignant or not and whether metastasis can be determined or not, and abdominal computed tomography has recently been reported to be the most useful method for the diagnosis of adult intussusception. In other reports, presurgical diagnoses were made by using CT in most cases $[1,3,6]$.

For the treatment of intussusception, even now, constant treatment trends have not been established; nonetheless, when a causative lesion is present and the possibility of malignant tumors is high, the treatments of choice is to perform surgery. It is controversial whether the invaginated intestine should be reduced prior to resection. In small bowel and right ascending colon intussusception cases, first, manual reduction is performed; the causative lesion is then assessed and treated. Cases in the small bowel develops complications and thus cannot be restored or transverse colon and descending colon intussusceptions are resected directly without resection because benign lesions are abundant in small intestine cases and the incidence of malignancy is high in the left colon. In addition, for cases in which perforation due to the excess manipulation during reduction or the dissemination of malignant cells is a worry, resection in a wide area should be performed [6, 16]. Recently, with the improvement of laparoscopic surgery, cases in which a laparoscopic anterior resection was performed after the reduction of the sigmoidorectal intussusception through the anus have been reported; nonetheless, successful laparoscopic treatments of intussusceptions caused by malignant tumors have been rarely reported, and studies on the treatment outcomes of laparotomic surgery for intussusceptions and its prognosis have not been reported [11]. If surgery is performed after the reduction of the invaginated area caused by malignant tumors, because of the possibility of necrosis of the mucosa during the reduction procedure, the dissemination of malignant cells due to perforation, and the venous embolism of malignant cells, prior reduction of herniation has not been accepted in most studies $[1,18]$. With the recent remarkable improvement in laparoscopic surgery, cases with intussusceptions cause by malignant tumors in the lower rectum treated by laparoscopic reduction first, followed by successful surgery, have been reported, although they are rare $[11,19,20]$. Adult intussusception caused by a malignant mucinous adenocarcinoma is a very rare disease. Furthermore, different from pediatric cases showing very specific symptoms or syndrome, the diagnosis of an adult sigmoidorectal intussusception caused by malignant tumors may be difficult, and on the digital rectal examination, it may be misdiagnosed as lower rectal cancer. Therefore, accurate presurgical diagnosis and appropriate surgery are required. Surgical treatments are a laparotomy, laparoscopic surgery, or a resection through the anus. It is thought that sigmoidorectal intussusception cases that might be misdiagnosed as lower rectal cancer as in our case may be treated by successful reduction of the intussusception by using an end-to-end anastomosis dilator and laparoscopy through the anus. However, the possibility of dissemination of malignant tumor cells caused by excessive manipulation and venous thrombi should be well understood and avoided. Based on the advantages of laparoscopic surgery, its cosmetic aspect, shortening of the hospitalization period, and early return to normal life, surgery on the lower rectal area could be avoided injury to the autonomic nervous system Therefore case for sigmoidorectal intussusception caused by tumors, the procedure of reduction first, followed by laparoscopic surgery may be considered.

In summary, we experienced the case of a patient admitted for the chief complaints of diarrhea and hematochezia. First, we reduced the sigmoidorectal intussusception which was caused by a mucinous adenocarcinoma that had developed in the sigmoid colon. Subsequently, we performed a laparoscopic anterior resection. This case was reported, together with a review of the literature.

\section{CONFLICT OF INTEREST}

No potential conflict of interest relevant to this article was reported.

\section{ACKNOWLEDGEMENT}

This research supported by Wonkwang University, 2009.

\section{REFERENCES}

1. Azar T, Berger DL. Adult intussusception. Ann Surg 1997;226: 134-8.

2. Weilbaecher D, Bolin JA, Hearn D, Ogden W 2nd. Intussusception in adults: review of 160 cases. Am J Surg 1971;121:531-5.

3. Dujardin M, de Beeck BO, Osteaux M. Inverted Meckel's diverticulum as a leading point for ileoileal intussusception in an adult: case report. Abdom Imaging 2002;27:563-5.

4. Matsuba Y, Mizuiri H, Murata T, Niimi K. Adult intussusception due to lymphangioma of the colon. J Gastroenterol 2003;38:181-5.

5. Nagorney DM, Sarr MG, McIlrath DC. Surgical management of intussusception in the adult. Ann Surg 1981;193:230-6.

6. Begos DG, Sandor A, Modlin IM. The diagnosis and management of adult intussusception. Am J Surg 1997;173:88-94.

7. Matsuda K, Suda K, Tamura K, Deguchi T, Yamazaki E, Yago H, 
et al. Surgical management of adult sigmoid colon intussusception caused by a malignant tumor: report of a case. Surg Today 2003;33:768-71.

8. Warshauer DM, Lee JK. Adult intussusception detected at CT or MR imaging: clinical-imaging correlation. Radiology 1999;212: 853-60.

9. Hutchinson J. A successful case of abdominal section for intussusception. Proc R Med Chir Soc 1873;7:195-8.

10. Rosière A, de Canniere L, Frangi R, Michel LA. Early diagnosis of adult intestinal intussusception at four different locations. Acta Chir Belg 1994;94:314-7.

11. Park KJ, Choi HJ, Kim SH, Han SY, Hong SH, Cho JH, et al. Sigmoidorectal intussusception of adenoma of sigmoid colon treated by laparoscopic anterior resection after sponge-on-the-stick-assisted manual reduction. World J Gastroenterol 2006;12:146-9.

12. Park YJ. Adult sigmoido-recto-anal intussusception by a sigmoid colon adenoma. J Korean Soc Coloproctol 2009;25:121-4.

13. Kanemitsu Y, Kato T, Hirai T, Yasui K, Morimoto T, Shimizu Y, et al. Survival after curative resection for mucinous adenocarcinoma of the colorectum. Dis Colon Rectum 2003;46:160-7.

14. Du W, Mah JT, Lee J, Sankila R, Sankaranarayanan R, Chia KS. Incidence and survival of mucinous adenocarcinoma of the colorectum: a population-based study from an Asian country. Dis Colon Rectum 2004;47:78-85.

15. Lorigan JG, DuBrow RA. The computed tomographic appearances and clinical significance of intussusception in adults with malignant neoplasms. Br J Radiol 1990;63:257-62.

16. Brayton D, Norris WJ. Intussusception in adults. Am J Surg 1954; 88:32-43.

17. Boyle MJ, Arkell LJ, Williams JT. Ultrasonic diagnosis of adult intussusception. Am J Gastroenterol 1993;88:617-8.

18. Eisen LK, Cunningham JD, Aufses AH Jr. Intussusception in adults: institutional review. J Am Coll Surg 1999;188:390-5.

19. Dean DL, Ellis FH, Sauer WG. Intussusception in adults. AMA Arch Surg 1956;73:6-11.

20. Chuang Ch, Hsieh C, Lin Ch, Yu J. Laparoscopic management of sigmoid colon intussusception caused by a malignant tumor: case report. Rev Esp Enferm Dig 2007;99:615-6. 